\title{
Visualisations of Centre Vortices
}

\author{
James Biddle ${ }^{1, *}$, Waseem Kamleh ${ }^{1, * *}$, and Derek Leinweber ${ }^{1, * * *}$ \\ ${ }^{1}$ Centre for the Subatomic Structure of Matter, Department of Physics, The University of Adelaide, SA \\ 5005, Australia
}

\begin{abstract}
The centre vortex structure of the vacuum is visualised through the use of novel $3 \mathrm{D}$ visualisation techniques. These visualisations allow for a hands-on examination of the centre-vortex matter present in the QCD vacuum, and highlights some of the key features of the centre-vortex model. The connection between topological charge and singular points is also explored. This work highlights the useful role visualisations play in the exploration of the QCD vacuum.
\end{abstract}

\section{Introduction}

Our current understanding of the strong interaction is encapsulated in the gauge field theory of Quantum Chromodynamics (QCD). Because the gauge bosons of QCD, the gluons, can selfinteract, the QCD vacuum is populated by highly non-trivial gluon and quark condensates. However, it is not yet analytically determined what feature of the non-trivial QCD ground state fields is fundamental to the distinctive properties of QCD, namely the

- Confinement of quarks, and

- Dynamical chiral symmetry breaking leading to dynamical mass generation.

The most promising candidate supported by numerical studies is the centre vortex picture $[1,2]$, which postulates that these two features are caused by sheets of chromo-magnetic flux carrying charge associated with the centre of the $S U(3)$ gauge group, given by the three values of $\sqrt[3]{1}$. The centre vortex picture has already had much success in reproducing many distinctive QCD properties, such as the linear static quark potential [3-7], enhancement of the infrared gluon propagator [8-11], enhancement of the infrared quark mass function $[12,13]$ and mass splitting in the low-lying hadron spectrum [13-15].

This work seeks to visualise these centre vortices on the lattice through the use of 3D modelling techniques, allowing us to explore the vortex vacuum in a never-before-seen way. These visualisations are presented as interactive 3D models embedded in the document. To interact with these models, it is necessary to open the document in Adobe Reader or Adobe Acrobat (requires version 9 or newer). Linux users should install Adobe Acroread version 9.4.1, the last edition to have full 3D support. Note that 3D content must also be enabled for the interactive content to be available, and for proper rendering it is necessary to enable

\footnotetext{
*e-mail: james.biddle@adelaide.edu.au

**e-mail: waseem.kamleh@adelaide.edu.au

***e-mail: derek.leinweber@adelaide.edu.au
} 

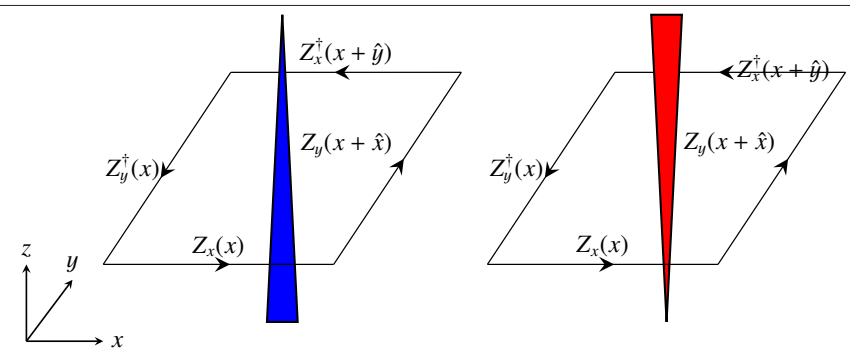

Figure 2. An example of the plotting convention for vortices located within a 3D time slice. Left: A +1 vortex in the $+\hat{z}$ direction. Right: $A-1$ vortex in the $-\hat{z}$ direction.

double-sided rendering in the preferences menu. To view the models, click on the figures marked as Interactive in the caption. To rotate the model, click and hold the left mouse button and move the mouse. Use the scroll wheel or shift-click to zoom. Some pre-set views of the model are also provided to highlight areas of interest. To reset the model back to its original orientation and zoom, press the 'home' icon in the toolbar or change the view to 'Default view'. In addition, the 3D models presented here can be viewed in augmented reality through Josh Charvetto's Android application. See Fig 1 for a QR code link to download the app.

\section{Spatially-Oriented Centre Vortices}

When projected onto 3D space, vortices appear as closed lines carrying centre charge. They are identified on the lattice by projecting the gluon field links onto their nearest centre element in maximal centre gauge. Each $1 \times 1$ Wilson loop, $P_{\mu \nu}(x)$, will then take one of three possible values

$$
P_{\mu v}(x)=\exp \left(\frac{m 2 \pi i}{3}\right) I, m \in\{-1,0,+1\} .
$$

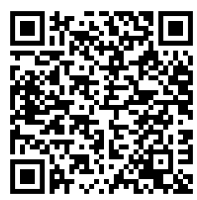

If $P_{\mu \nu}(x)$ takes one of the two complex phases, we say it is pierced by a vortex. We refer to the vortex by it's centre charge parameter, $m= \pm 1$.

For a charge $m=+1$ vortex, a blue jet is plotted positively oriented piercing the centre of the plaquette, and for a charge $m=-1$ vortex, a red jet is plotted negatively oriented. An example of this convention is shown in Fig. 2. With these conventions, the first time slice of a gluon field configuration appears as illustrated in Fig. 3. From this visualisation we note a couple of interesting properties. Firstly, vortices must form closed lines to conserve centre flux. However, a vortex line is permitted to branch into 2 lines due to the periodic property of the centre group. These features are highlighted in Fig. 4. We also note that vortex loops tend to be large, which is indicative of the confining phase [16].

\section{Space-Time Oriented Vortices}

In $4 \mathrm{D}$ space-time, centre vortices map out a $2 \mathrm{D}$ wold sheet. To visualise these vortices, we project onto 3D space where the sheets map out lines that vary with time. As we have taken 


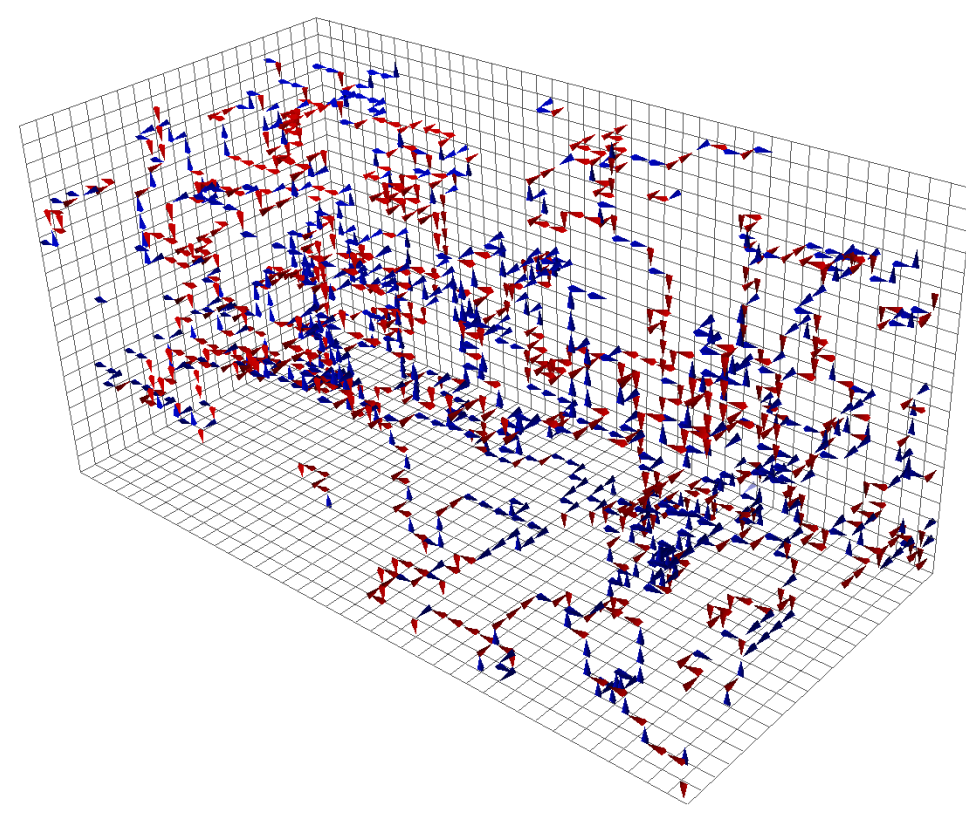

Figure 3. The first time-slice of spatially-oriented vortices. (Interactive online)
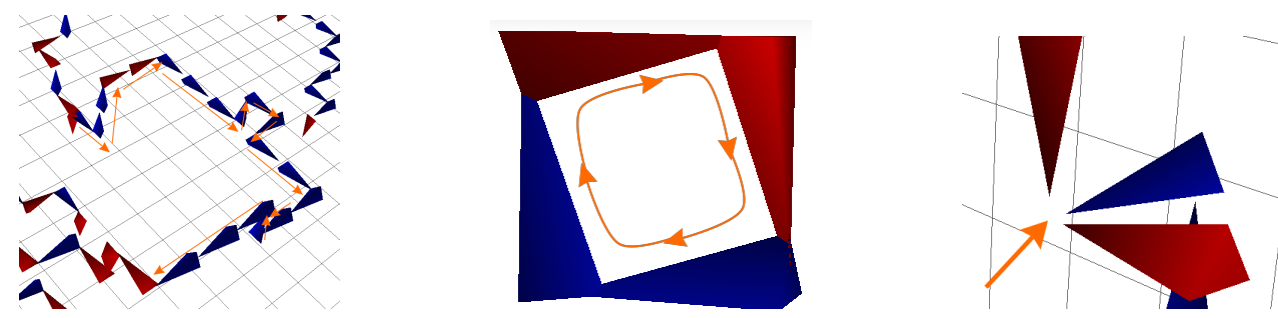

Figure 4. Left: Vortices form continuous lines, highlighted with orange arrows in this diagram. Middle: Vortices must form closed loops to conserve the vortex flux. Right: $S U(3)$ vortices are capable of forming monopoles or branching points where three vortices emerge or converge at a single point.

3D slices, we have suppressed all vortex information in the time direction. In each 3D slice we only have access to one link belonging to the plaquettes associated with vortices in the forwards and backwards $x_{i}-t$ planes. As such we plot an 'indicator' link, to signify the presence of a vortex in the suppressed direction. This follows the convention,

- +1 vortex, forward in time $\Longrightarrow$ cyan arrow, positively oriented

- +1 vortex, backward in time $\Longrightarrow$ cyan arrow, negatively oriented

- -1 vortex, forward in time $\Longrightarrow$ orange arrow, positively oriented

- -1 vortex, backward in time $\Longrightarrow$ orange arrow, negatively oriented.

An example of these conventions is shown in Fig. 5. Adding these space-time indicator links 

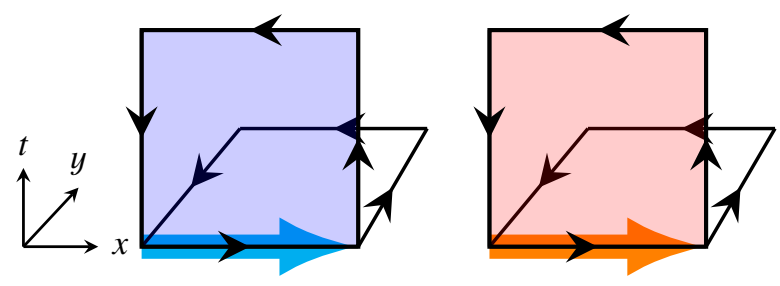

Figure 5. Left: $\mathrm{A}+1$ vortex in the forward $x-t$ plane (shaded blue) will be indicated by a cyan arrow in the $+\hat{x}$ direction. Right: A -1 vortex in the forward $x-t$ plane (shaded red) will be indicated by an orange arrow in the $+\hat{x}$ direction.

to our previous visualisation, the first time slice now appears as Fig. 6.

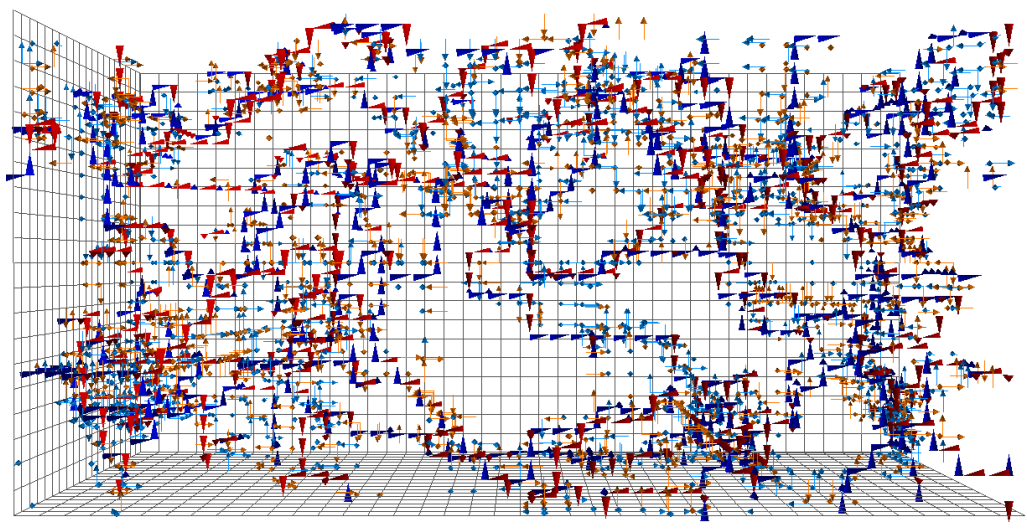

Figure 6. The first time-slice of now containing both spatially-oriented and space-time vortices. (Interactive online)

We can see how these space-time oriented indicator links predict the motion of spatiallyoriented vortices by looking at Fig. 7. Here we see a line of $m=-1$ vortices shifting along the sheet of cyan space-time oriented indicator links as we step through time.

\section{Singular Points}

Using our vortex illustrations, we can identify locations where vortex surfaces span all four space-time dimensions. These locations are known as singular points, and can be identified as shown below by an indicator link running parallel to a spatially-oriented jet, as shown in Fig. 8. These points are significant because they necessarily generate regions of topological charge density. A visualisation of these singular points is presented in Fig. 9. 


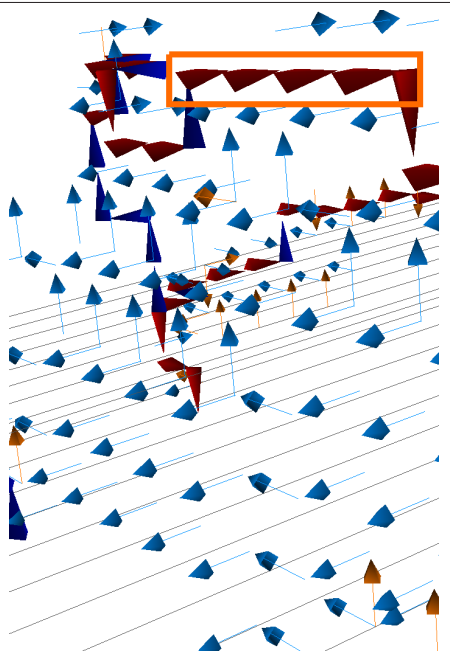

(a) $t=1$

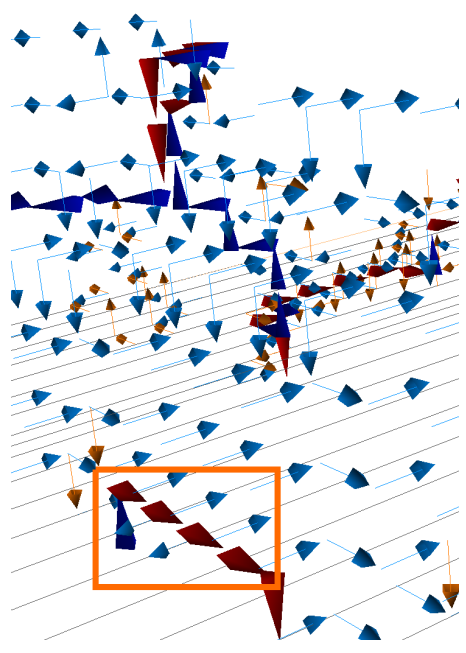

(b) $t=2$

Figure 7. An example of a sheet of space-time oriented vortices predicting the motion of spatiallyoriented vortices over multiple lattice sites from $t=1$ to $t=2$.

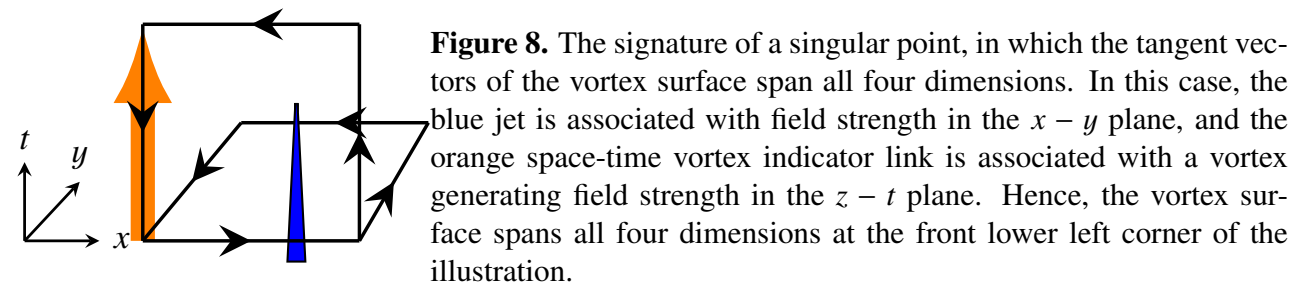

Figure 8. The signature of a singular point, in which the tangent vectors of the vortex surface span all four dimensions. In this case, the blue jet is associated with field strength in the $x-y$ plane, and the orange space-time vortex indicator link is associated with a vortex generating field strength in the $z-t$ plane. Hence, the vortex surillustration.

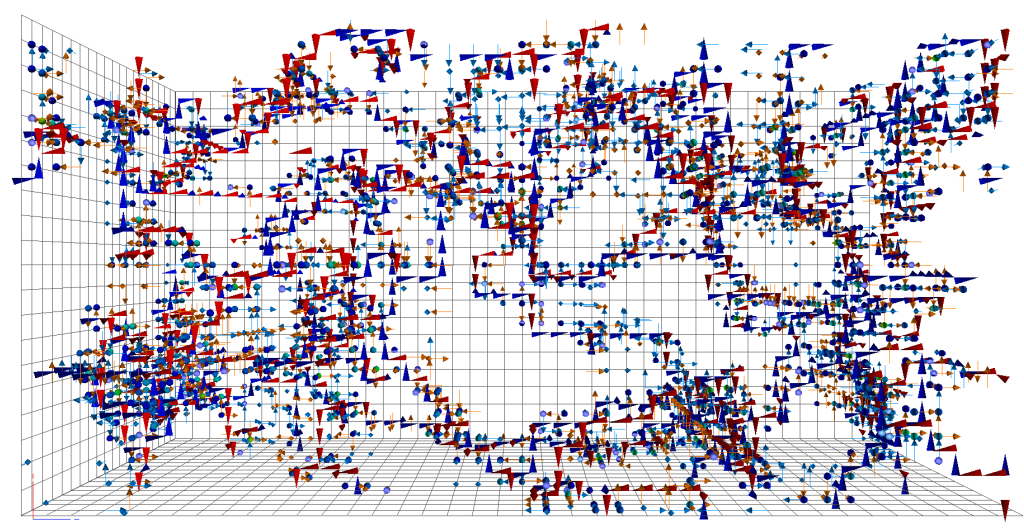

Figure 9. The singular points on the $t=1$ time slice presented in Fig. 6 are identified by spheres. (Interactive online) 


\section{Conclusion}

Visualisations of centre vortices provide valuable insight into the nature of the QCD vacuum. Through these visualisations we can identify structures of interest such as branching points and singular points, and study their relationship with topological charge density. For a more detailed description of these visualisations, and an analysis of the correlation between vortices and topological charge, see Ref. [17]. Work such as this allows one to explore QCD and centre vortices in a novel manner, and provides exciting new perspectives on the centre vortex vacuum.

\section{Acknowledgments}

The authors wish to thank Amalie Trewartha for her contributions to the gauge ensembles underlying this investigation. We also thank Ian Curington of Visual Technology Services Ltd. for his support of the PDF3D software used in creating the interactive 3D visualisations presented herein. This research is supported with supercomputing resources provided by the Phoenix HPC service at the University of Adelaide and the National Computational Infrastructure (NCI) supported by the Australian Government. This research is supported by the Australian Research Council through Grants No. DP190102215, DP150103164, DP190100297 and LE190100021.

\section{References}

[1] G. 't Hooft, Nucl.Phys. B138, 1 (1978)

[2] G. 't Hooft, Nucl.Phys. B153, 141 (1979)

[3] A. O'Cais, W. Kamleh, K. Langfeld, B. Lasscock, D. Leinweber, P. Moran, A. Sternbeck, L. von Smekal, Phys. Rev. D82, 114512 (2010), 0807.0264

[4] K. Langfeld, Phys. Rev. D69, 014503 (2004), hep-lat/0307030

[5] D. Trewartha, W. Kamleh, D. Leinweber, Phys. Rev. D92, 074507 (2015), 1509.05518

[6] J. Greensite, Prog. Part. Nucl. Phys. 51, 1 (2003), hep-lat/0301023

[7] L. Del Debbio, M. Faber, J. Giedt, J. Greensite, S. Olejnik, Phys. Rev. D58, 094501 (1998), hep-lat/9801027

[8] P.O. Bowman, K. Langfeld, D.B. Leinweber, A. Sternbeck, L. von Smekal, A.G. Williams, Phys. Rev. D84, 034501 (2011), 1010.4624

[9] J.C. Biddle, W. Kamleh, D.B. Leinweber, Phys. Rev. D98, 094504 (2018), 1806.04305

[10] K. Langfeld, H. Reinhardt, J. Gattnar, Nucl. Phys. B621, 131 (2002), hep-ph/0107141

[11] M. Quandt, H. Reinhardt, G. Burgio, Phys. Rev. D81 (2010), 1001. 3699

[12] P.O. Bowman, K. Langfeld, D.B. Leinweber, A. O' Cais, A. Sternbeck, L. von Smekal, A.G. Williams, Phys. Rev. D78, 054509 (2008), 0806. 4219

[13] D. Trewartha, W. Kamleh, D. Leinweber, Phys. Lett. B747, 373 (2015), 1502 . 06753

[14] D. Trewartha, W. Kamleh, D. Leinweber, J. Phys. G44, 125002 (2017), 1708.06789

[15] E.A. O’Malley, W. Kamleh, D. Leinweber, P. Moran, Phys. Rev. D86, 054503 (2012), 1112.2490

[16] M. Engelhardt, K. Langfeld, H. Reinhardt, O. Tennert, Phys. Rev. D61, 054504 (2000), hep-lat/9904004

[17] J.C. Biddle, W. Kamleh, D.B. Leinweber (2019), 1912 . 09531 